University of Wollongong

Research Online

Faculty of Engineering and Information

Faculty of Engineering and Information

Sciences - Papers: Part A

Sciences

$1-1-2016$

Design and development of a parametrically excited nonlinear energy harvester

Tanju Yildirim

University of Wollongong, ty370@uowmail.edu.au

Mergen H. Ghayesh

University of Adelaide, mergen.ghayesh@adelaide.edu.au

Weihua Li

University of Wollongong, weihuali@uow.edu.au

Gursel Alici

University of Wollongong, gursel@uow.edu.au

Follow this and additional works at: https://ro.uow.edu.au/eispapers

Part of the Engineering Commons, and the Science and Technology Studies Commons

Research Online is the open access institutional repository for the University of Wollongong. For further information contact the UOW Library: research-pubs@uow.edu.au 


\title{
Design and development of a parametrically excited nonlinear energy harvester
}

\begin{abstract}
An energy harvester has been designed, fabricated and tested based on the nonlinear dynamical response of a parametrically excited clamped-clamped beam with a central point-mass; magnets have been used as the central point-mass which pass through a coil when parametrically excited. Experiments have been conducted for the energy harvester when the system is excited (i) harmonically near the primary resonance; (ii) harmonically near the principal parametric resonance; (iii) by means of a nonsmooth periodic excitation. An electrodynamic shaker was used to parametrically excite the system and the corresponding displacement of the magnet and output voltages of the coil were measured. It has been shown that the system displays linear behaviour at the primary resonance; however, at the principal parametric resonance, the motion characteristic of the magnet substantially changed displaying a strong softening-type nonlinearity. Theoretical simulations have also been conducted in order to verify the experimental results; the comparison between theory and experiment were within very good agreement of each other. The energy harvester developed in this paper is capable of harvesting energy close to the primary resonance as well as the principal parametric resonance; the frequency-band has been broadened significantly mainly due to the nonlinear effects as well as the parametric excitation.
\end{abstract}

\section{Keywords}

nonlinear, design, energy, development, harvester, parametrically, excited

\section{Disciplines}

Engineering | Science and Technology Studies

\section{Publication Details}

Yildirim, T., Ghayesh, M. H., Li, W. \& Alici, G. (2016). Design and development of a parametrically excited nonlinear energy harvester. Energy Conversion and Management, 126 247-255. 


\title{
Design and development of a parametrically excited nonlinear energy harvester
}

\author{
Tanju Yildirim ${ }^{\text {a }}$, Mergen H. Ghayesh ${ }^{\text {b }}$, Weihua Li $^{\text {a }}$, Gursel Alici ${ }^{\text {a }}$ \\ ${ }^{a}$ School of Mechanical, Materials and Mechatronics Engineering, University of Wollongong, Northfields \\ Avenue, NSW 2522, Australia \\ ${ }^{b}$ School of Mechanical Engineering, University of Adelaide, South Australia 5005, Australia
}

Abstract

An energy harvester has been designed, fabricated and tested based on the nonlinear dynamical response of a parametrically excited clamped-clamped beam with a central pointmass; magnets have been used as the central point-mass which pass through a coil when parametrically excited. Experiments have been conducted for the energy harvester when the system is excited (i) harmonically near the primary resonance; (ii) harmonically near the principal parametric resonance; (iii) by means of a non-smooth periodic excitation. An electrodynamic shaker was used to parametrically excite the system and the corresponding displacement of the magnet and output voltages of the coil were measured. It has been shown that the system displays linear behaviour at the primary resonance; however, at the principal parametric resonance, the motion characteristic of the magnet substantially changed displaying a strong softening-type nonlinearity. Theoretical simulations have also been conducted in order to verify the experimental results; the comparison between theory and experiment were within very good agreement of each other. The energy harvester developed in this paper is capable of harvesting energy close to the primary resonance as well as the principal parametric resonance; the frequency-band has been broadened significantly mainly due to the nonlinear effects as well as the parametric excitation. 


\section{Introduction}

Due to increased demands for energy and current limitations of batteries, a future prospective technology is motion based energy harvesters (MBEHs) that convert kinetic energy into electrical energy [1]; this type of energy harvester has the potential to be used in powering electronic devices in hostile or remote environments — another benefit of MBEH devices is that they reduce pollutants in the environment which are left behind when batteries are disposed.

For the transduction mechanisms used to convert kinetic energy into electrical power; these can be grouped into three main categories. The first category uses piezoelectric conversion, which converts mechanical strain into electrical energy; for instance, Adhikari et al. [2] numerically investigated motion based energy harvesting under broadband excitation using a stack configuration of piezoelectric energy harvesters-expressions were derived for the non-dimensional time constant, electromechanical coupling coefficient and viscous damping factor. Renno et al. [3] investigated into the optimised power that can be harvested using a piezoelectric converter; using the Karush-Kuhn-Tucker technique, the power can be substantially enhanced by using an optimal inductor in the load circuit. Fan et al. [4] numerically and experimentally investigated the performance of a bi-directional nonlinear piezoelectric energy harvester; to achieve a nonlinear frequency-voltage curve two embedded magnets were used inducing a nonlinear stiffness-results showed that the bi-directional energy harvester was more effective than its linear counterpart. Fan et al. [5] designed a piezoelectric based energy harvester that could effectively harvest energy from sway and bi- 
directional motions; using a cantilever beam, frame and roller a frequency-up conversion mechanism was also achieved — results showed that the output voltage could be enhanced by increasing the sway frequency. Guan et al. [6] recently developed a piezoelectric energy harvester for rotational motion; a theoretical model was developed for the power output and experimental results showed good agreement with the theory. The second category of ambient motion energy harvesting transduction mechanisms are electrostatic conversion, where capacitive plates fluctuate inducing a voltage; for example, $\mathrm{Bu}$ et al. [7] fabricated a non-resonant wideband micro-energy harvester using electrostatic conversion- to boost low frequency excitations, a parallel structure was used to double the output voltage. The third mechanism that can be used to convert ambient kinetic energy into electrical power is electromagnetic induction (EMI), where the relative motion between a magnet and coil generates a backward electromotive force (EMF). For instance, Sardini et al. [8] developed a low frequency energy harvester using polymeric material, due to their low Young's modulus; a theoretical and experimental investigation was conducted-results showed that a small increase in bandwidth could be achieved with this design. Marin et al. [9] fabricated a linear electromagnetic energy harvester with a four Hertz bandwidth using four cantilever beams with closely related natural frequencies. Ooi et al. [10] designed a novel wideband electromagnetic energy harvester using dual resonating cantilever beams; a numerical model was done using MATLAB-Simulink; dual energy harvested peaks were observed resulting in a slight increase in the devices bandwidth. Recently, Siddique [11] presented a comprehensive review of micro-power generators using electromagnetic and piezoelectric energy harvesters; a significant review of recent motion based energy harvesters were compared to each other.

A major drawback with conventional MBEHs is the effective operating frequency range which energy can be harvested; in practical applications, where the excitation 
frequency is changing or varying with time, precisely matching the natural frequency of a device is crucial for operation and can be achieved with tuning techniques, however, the power required to tune a device will never result in greater energy harvested [12]. There are two main classes for MBEH devices based on the core element being used; the first class employs linear resonators and the second class uses nonlinear resonators.

For the first class, i.e. the linear energy harvesters, the maximum energy harvested is achieved when the excitation frequency matches the primary natural frequency of the core element in the MBEH device. The literature regarding this class is quite large. For instance, William and Yates [13] first investigated the use of external resonating devices for powering micro electrical mechanical systems (MEMS); this work was further theoretically extended by Mitcheson et al. [14] for three different damping based resonators. Stephen [15] theoretically investigated the potential of linear $\mathrm{MBEH}$ devices for direct mass and base excitations, including the coupling between mechanical and electrical domains. Shahruz [16] developed a multimodal array based on transversely excited cantilever beams with different geometries and tip masses; the results showed that the combination of these linear energy harvesters could achieve a larger bandwidth, however, the required circuitry was more complex. Tang and Zuo [17] theoretically analysed dual mass linear resonators to widen the bandwidth of MBEH devices; dual mass devices could have two local optimums which can further increase the bandwidth of the device. Erturk and Inman [18] further investigated transversely excited linear cantilever beams with piezoelectric bimorph layers, using EulerBernoulli beam theory for use as an energy harvester. Leland and Wright [19] designed a linear MBEH device that could be tuned with compressive axial preloading further extending the operating bandwidth of the energy harvester; other tuneable linear resonators based on preloading mechanisms for energy harvesters have also been developed for example in [20]. 

harvesters whose core elements are either transversely excited or parametrically excited; for the transversely excited system, there is a large volume of available literature. For instance, Mann and Simms [21] fabricated a nonlinear energy harvester based on the transverse oscillation of a levitated magnet; a perturbation technique known as the method of multiple scales was employed to derive the theoretical frequency response curve-experimental and theoretical results were within good agreement. Maiorca et al. [22] and Liu et al. [23] fabricated $\mathrm{MBEH}$ devices utilising mechanical stoppers in which both devices showed nonlinear energy extraction near the primary resonance. Sebald et al. [24] analysed the effects of magnetically induced nonlinearities in transversely excited cantilever beams with piezoelectric layers experimentally. In general, bi-stable energy harvesters have a broader bandwidth [25] and the energy harvested is not influenced under white noise [26]. energy harvesters based on parametric excitations of their core elements, is not extensive; for example, Abdelkefi et al. [27] theoretically investigated a parametrically excited cantilever beam for energy harvesting purposes using the Galerkin discretisation and the method of softening-type nonlinearity in the vicinity of the principal parametric resonance.

In this paper, for the first time, an energy harvester has been developed and tested experimentally based on the nonlinear dynamical behaviour of a parametrically excited beam carrying a point-mass as the core element subject to a magnetic field. The experiments showed that the fabricated device has an extended bandwidth for effectively harvesting 
kinetic energy; in particular, at the principal parametric resonance, the device displays a strong softening-type nonlinearity at higher frequencies which is used to further maximise the frequency band-width and hence kinetic energy harvested from the device- the device harvests energy at both the primary and principal-parametric resonances. The energy harvester designed based on parametric excitation is shown to harvest energy over larger frequency bands due to the qualitative and quantitative changes in the nonlinear dynamical behaviour of the core element. The paper has been organised as follows: a description of the system including the fabricated device, background theory and experimental procedure are developed in Section 2; the experimentally obtained results for the fabricated energy harvester are acquired and discussed in detail in Section 3. Theoretical verifications are provided in Section 4. Section 5 ends with concluding remarks.

\section{System description and experimental procedure}

This section describes the specifications of the energy harvester fabricated using a parametrically excited beam carrying a concentrated mass as the core element as well as the experimental setup and data recording and analysing system.

\subsection{System description}

The system shown in Fig. 1 is the core element of an energy harvester with a parametrically excited beam carrying a point-mass as the core element; one of the clamps is fixed while the other is a moveable support in the longitudinal direction. An aluminium beam with length $L$, width $b$ and thickness $h$ has dimensions of 160,12,0.6mm, respectively; a magnet has been attached to either side of the centre of the beam with a net weight of 0.0196 $\mathrm{kg}(m)$ (as the concentrated mass). A coil was used as the transduction method to convert the dynamic motion into the electrical energy through electromagnetic induction (EMI); the coil 
consisted of 600 tightly wound turns and had an internal resistance of 2.7 Ohms. The opencircuit voltage was measured during the experiments when the shaker was exciting the energy harvester (see Figure 2); the moveable support was achieved by using linear bearings.

\subsection{Theoretical background}

150

A parametrically excited perfect system displays a zero-response throughout the frequency space, the non-zero response emerges from the zero-response in the vicinity of the principal parametric resonance; however, an initial threshold is required to activate a parametric resonance - a characteristic parametric equation is known as the Mathieu equation given by

$\ddot{x}+\alpha x^{3}+[\omega-2 q \cos (2 t)] x=0$,

where $x$ is the displacement field, $\omega$ is the natural frequency, $q$ is the forcing amplitude, $\alpha$ is the nonlinear stiffness coefficient and $t$ is the time. Understanding Eq. (1) from a design perspectives means that energy can be harvested effectively from an imperfect system at two frequencies that are relatively close to each other (depending on system parameters); furthermore, the nonlinear stiffness term can be designed for using geometric extensibility at the centreline of the core element resulting in nonlinear behaviour and hence nonlinear frequency-response curves which will be further used to extend the operating bandwidth of the fabricated energy harvester. For the energy harvester shown in Fig.1, ambient kinetic energy is harvested using EMI; as the magnet passes through the coil an induced back electromotive force is generated in the coil resulting in the conversion of kinetic energy into electrical energy. The electromotive force generated is proportional to the rate of change in the magnetic flux given by [29] 
$169 \quad V_{E M F}=-\frac{d \phi}{d t}$,

where $V_{E M F}$ is the back electromotive force and $\phi$ is the magnetic field flux density. For a

171 uniform coil, the back EMF is proportional to the number of turns by [30]

$172 \quad V_{E M F}=-N \frac{d \phi}{d t}$,

(a)



(b)

$$
\mathrm{L} / 2
$$$$
\mathrm{L} / 2
$$




Figure 1: (a) A parametrically excited clamped-clamped beam carrying a concentrated mass as the core element of the energy harvester; (b) Schematic of (a)

\subsection{Experimental procedure}

Figure 2 shows the experimental setup and the connection between system components, 194 for measuring and acquiring experimental data for analysis. The beam was excited using an electrodynamic shaker (VTS, VC 100-8); the corresponding displacement of the central magnet was measured using a laser displacement sensor (Microepsilon opto NCDT1700ILD1700-50) and the output voltage of the coil was measured through a data acquisition board (DAQ National instruments PCI-6221). The closed loop control system was achieved using an accelerometer (CA YD106) connected to the electrodynamic shaker to control the base excitation amplitude; the output voltage of the DAQ board was enlarged through a power amplifier (Sinocera YE5871).

The system was excited in the horizontal plane and the resulting motion of the magnets was $90^{\circ}$ (perpendicular) to the base excitation; a swept sine signal in the form of $\mathrm{Y}=A \sin (\Omega t)$ was implemented to test the energy harvester. 


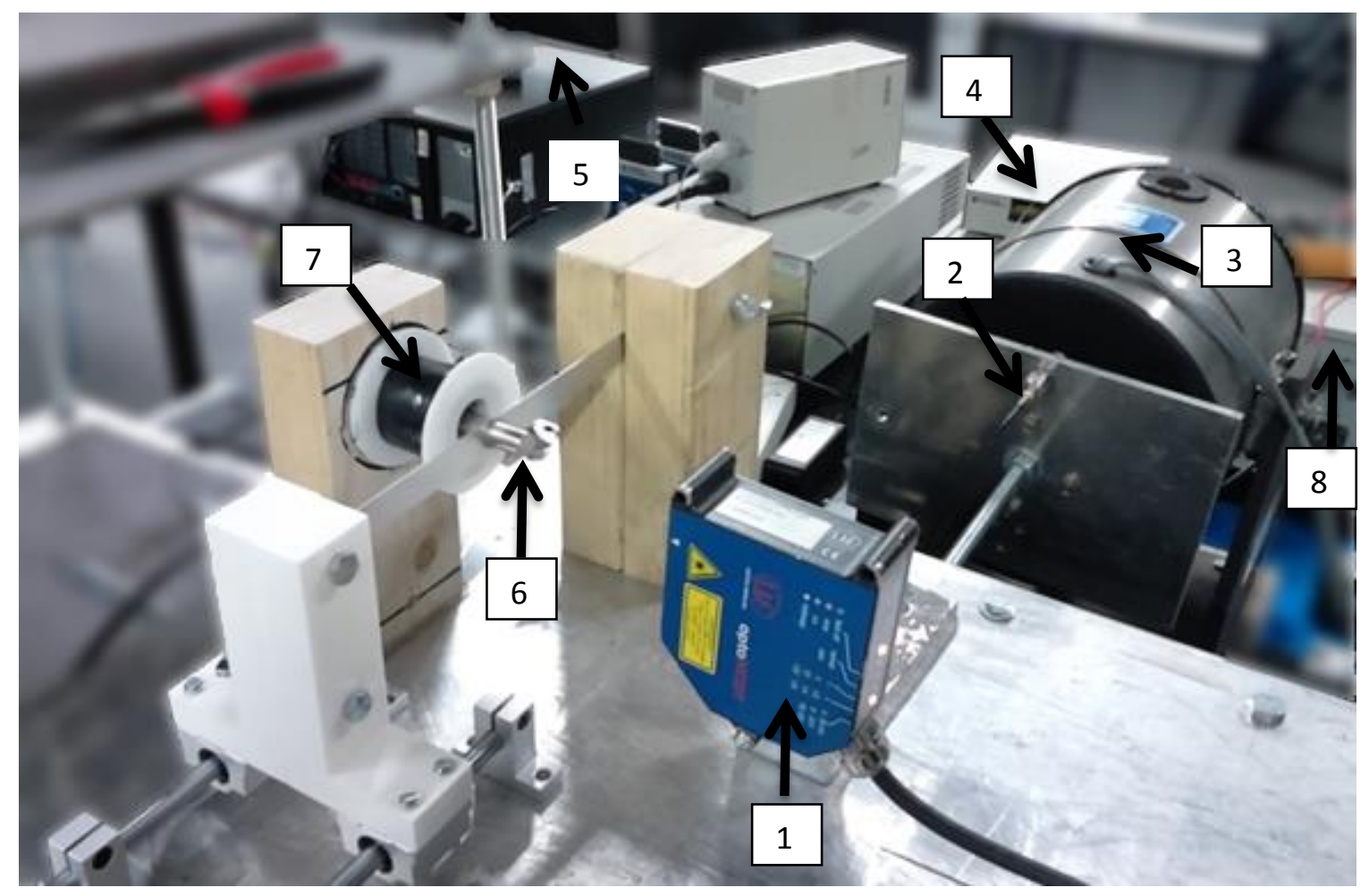

Figure 2: Photograph of the experimental setup (1. Laser Displacement Sensor; 2. Accelerometer; 3. Electrodynamic shaker; 4. DAQ board; 5. Computer; 6. Magnet; 7. Coil; 8. Power amplifier)

\section{Experimental results for energy harvested}

The fabricated energy harvester using a beam carrying a central mass as the core element was experimentally tested when the system was excited (i) harmonically near the primary resonance; (ii) harmonically near the principal resonance; (iii) near the principal resonance with a non-smooth periodic function. Experiments (i-iii) were used to qualitatively and quantitatively investigate the nonlinear dynamical response of the parametric resonator under different acceleration amplitudes to determine the MBEH devices broader operating range. A non-dimensional frequency has been introduced as the excitation frequency divided by the experimentally obtained fundamental primary linear resonance of the energy harvester $\left(\Omega / \omega_{1}\right)$ and at the principal parametric resonance $\left(\Omega / 2 \omega_{1}\right)$; similarly, a non-dimensional motion amplitude has been introduced as the parametric motion amplitude divided by the 
thickness of the beam (Displacement/h). The experimentally obtained results for the

222 displacement and voltage are presented; referring to Fig.2, the positive motion amplitude and voltage occur when the magnet is moving away from the displacement sensor and the negative motion amplitude and voltage occur when the magnet moves toward the displacement sensor. The frequency-response curves, time traces, fast Fourier transform (FFT), phase-plane diagrams and probability density function (PDF), for experiments (ii) and (iii) are experimentally obtained and plotted to describe the nonlinear dynamical response and hence the nonlinear energy harvested; the frequency-response curve for the open-circuit voltage of the energy harvester are also experimentally acquired and plotted.

\subsection{Energy harvested in the vicinity of the primary resonance}

In this section, experiments have been conducted in order to analyse the response of the energy harvester when excited in the vicinity of the primary resonance of the core element; results are plotted for both the energy harvested and motion characteristics of the resonator.

Figures 3(a)-(c) show the open-circuit voltage measured from the coil, the dimensioned displacement of the central mass and the non-dimensioned motion amplitude of the central mass, respectively. It was observed that there was a slight shift in the primary resonance at different excitation amplitudes; this was due to slight initial geometric imperfections in the beam - the corresponding primary resonance frequencies for $1,5.5,6.5$ $\mathrm{ms}^{-2}$ excitation amplitudes were experimentally obtained as 28.27, 28.12 and $27.95 \mathrm{~Hz}$, respectively. When the energy harvester was excited near the primary resonance, a linear response was observed characterised by a gradual incline to a maximum at $\Omega / \omega_{1}$ followed by a gradual and continuous decline; no jump phenomenon was observed. It was also shown in 
246 Fig.3 (a) that the open-circuit voltage was generated in the primary resonance region is 247 proportional to the relative displacement of the magnet and the coil.

(a)

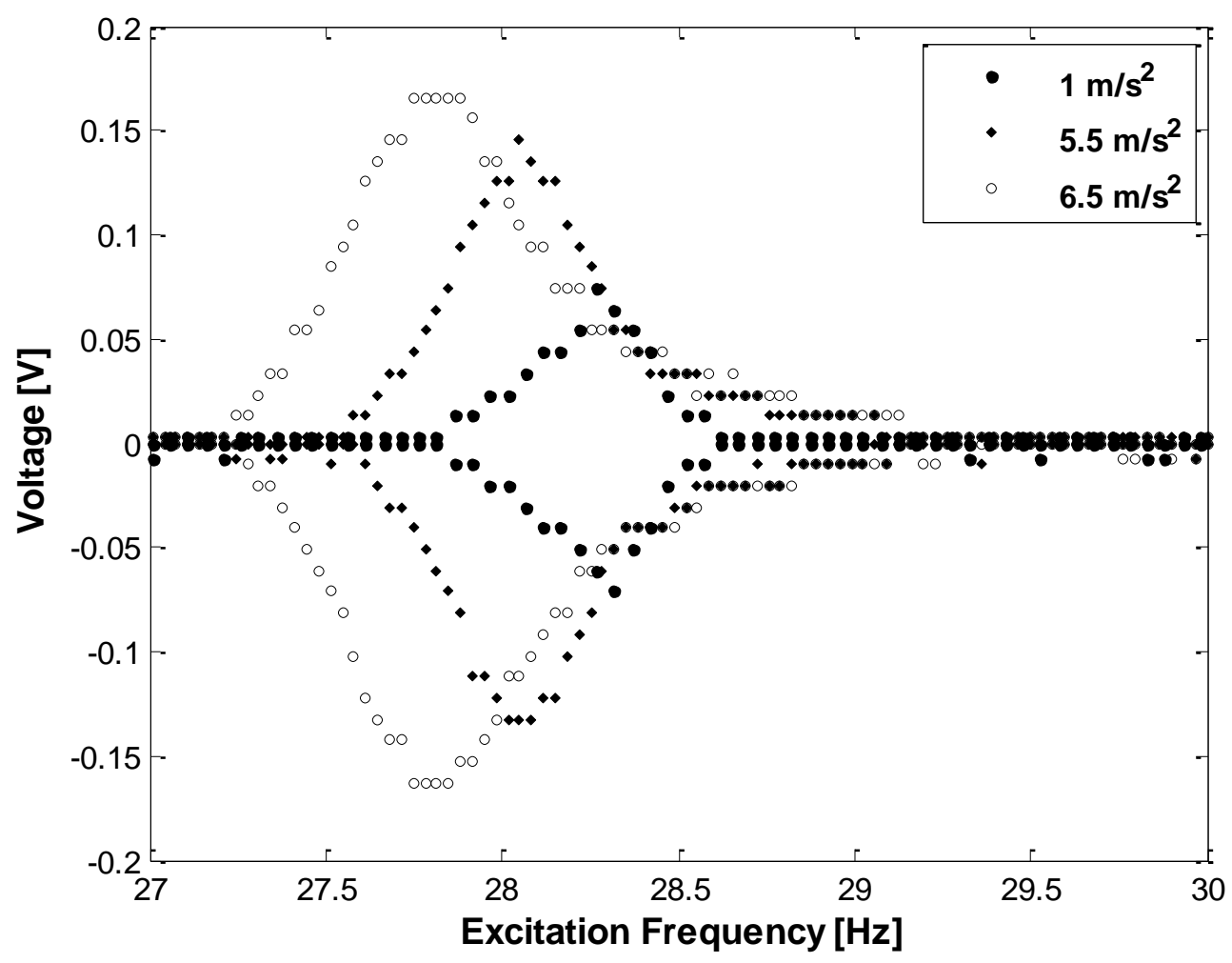


(b)

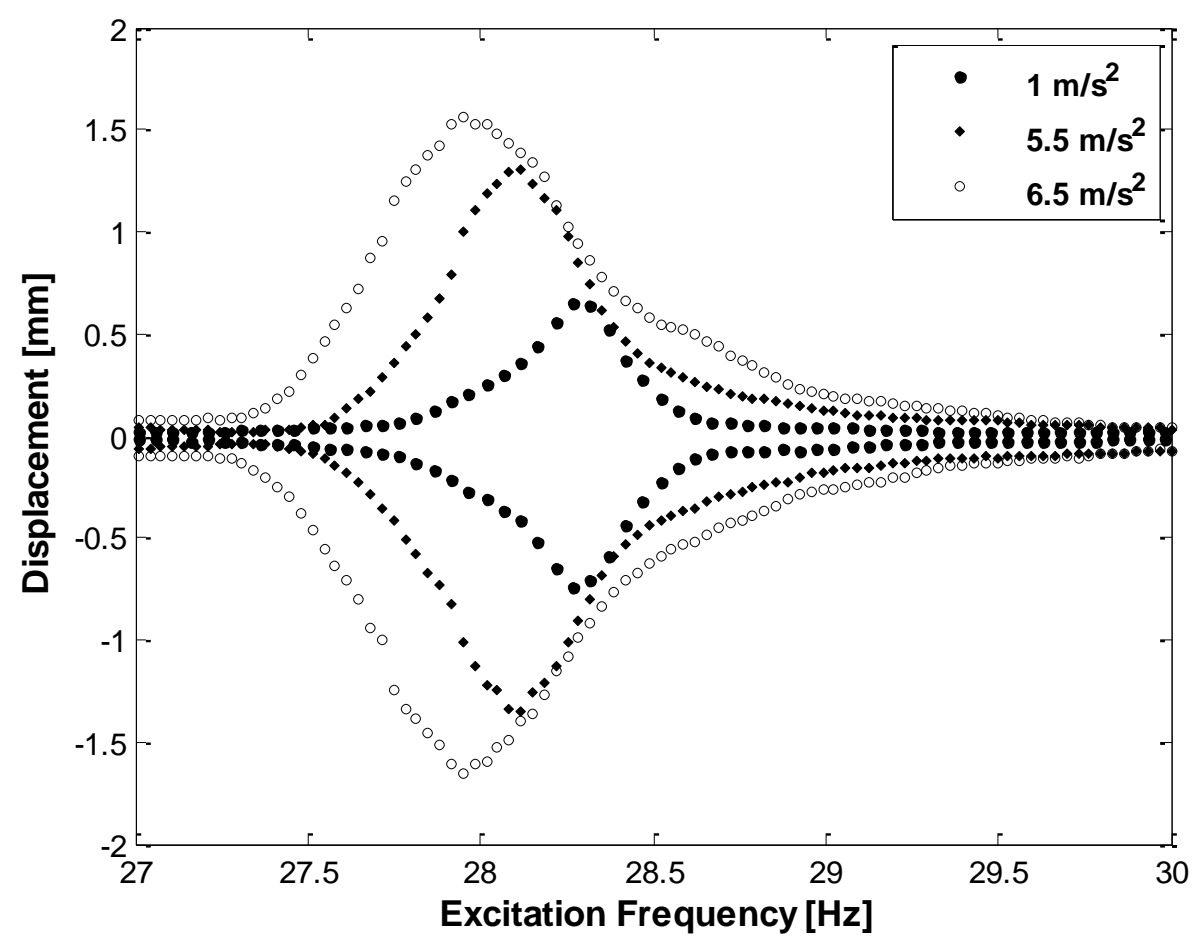

(c)

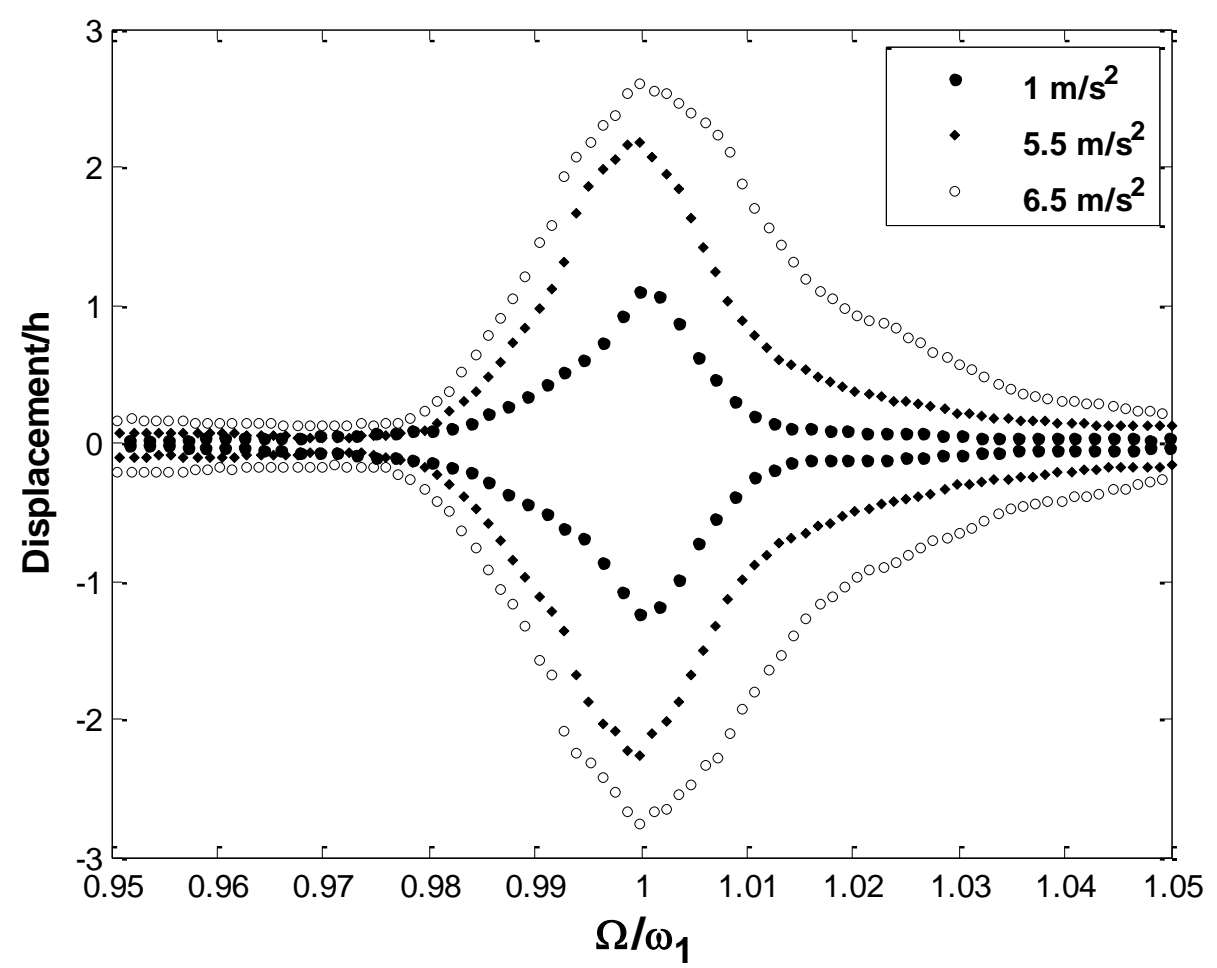




\subsection{Energy harvested in the vicinity of the principal parametric resonance}

In this section, the nonlinear energy harvested from the energy harvester is experimentally obtained when the nonlinear core element was excited in the vicinity of the principal parametric resonance; the experimentally obtained results were plotted in Fig. 4 and are discussed in the following.

It is shown in Fig. 4 that when the energy harvester is excited in the vicinity of the principal parametric resonance of the core element, the system displays a strong softeningtype nonlinearity bifurcated from a near-zero response; the softening behaviour can be explained due to initial geometric imperfections in the fabrication process which is realistic in manufacturing processes. Moreover, Fig. 4 displays a nonlinear dynamical behaviour characterised by having two discontinuous points, theoretically these discontinuous points are limit point and period-doubling bifurcations and experimentally these points occur as jump down and jump up points or sudden growth in the response; there are two responses at a specific excitation frequency which are the result of combing the forward and reverse frequency sweeps. The system shows substantially different energy harvested and motion characteristics compared to conventional linear energy harvesters in the literature, thus, requiring a nonlinear analysis. It is shown in Fig.4(a) that the nonlinear energy extracted in the vicinity of the principal parametric resonance improves the operating bandwidth of the energy harvester and the maximum energy harvested; it was observed that an $11.5 \%$ energy harvesting frequency band was achieved - it was also observed that there was a significant increase in the maximum open-circuit voltage by about $300 \%$, between, 5.5 and $6.5 \mathrm{~ms}^{-2}$ base excitation. For the maximum motion amplitude occurring at $54 \mathrm{~Hz}$ for $9 \mathrm{~ms}^{-2}$ excitation amplitude, the time trace, FFT, phase-plane diagram and PDF are shown in Figs.5(a)-(d), 
275 respectively, showing a period-2 motion; however, the system is slightly non-symmetric 276 which is due to the initial geometric imperfection.

(a)

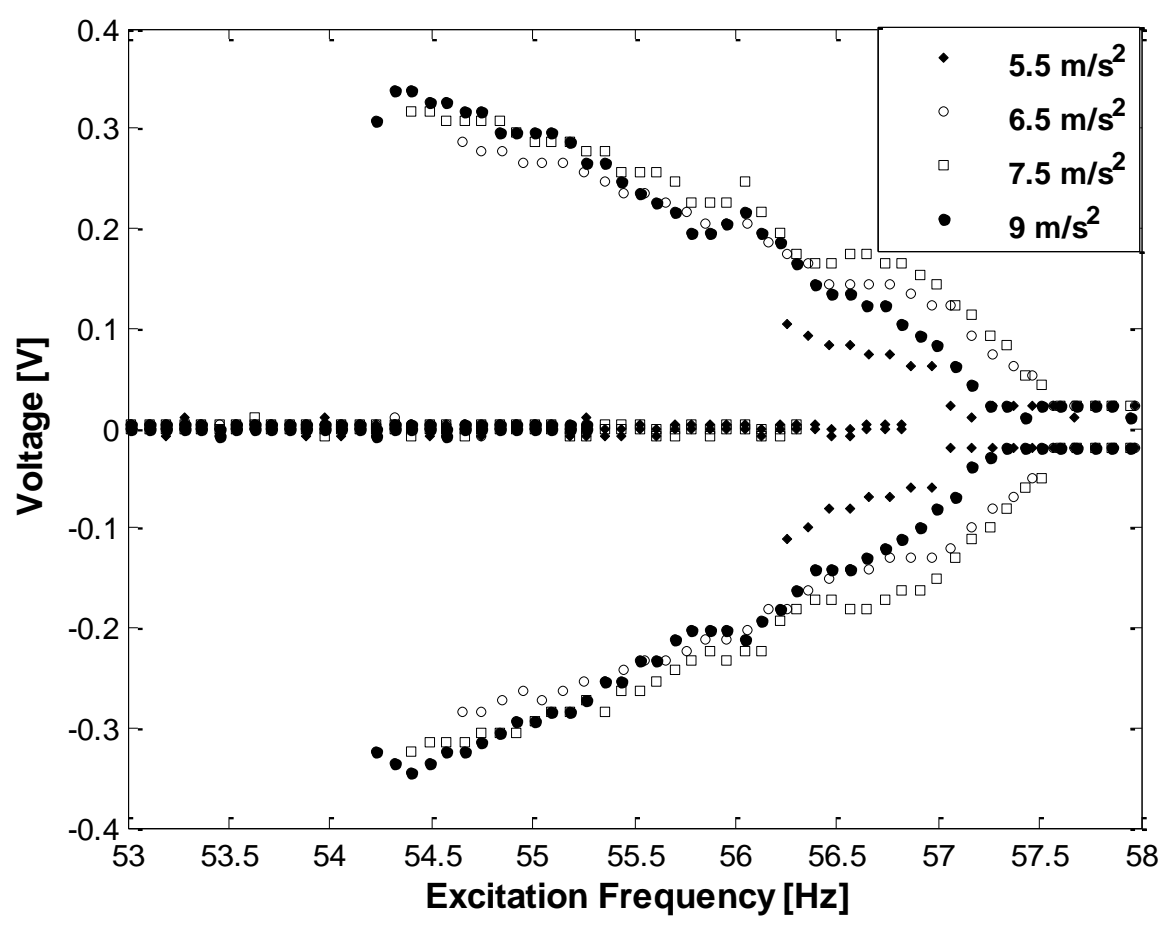


(b)

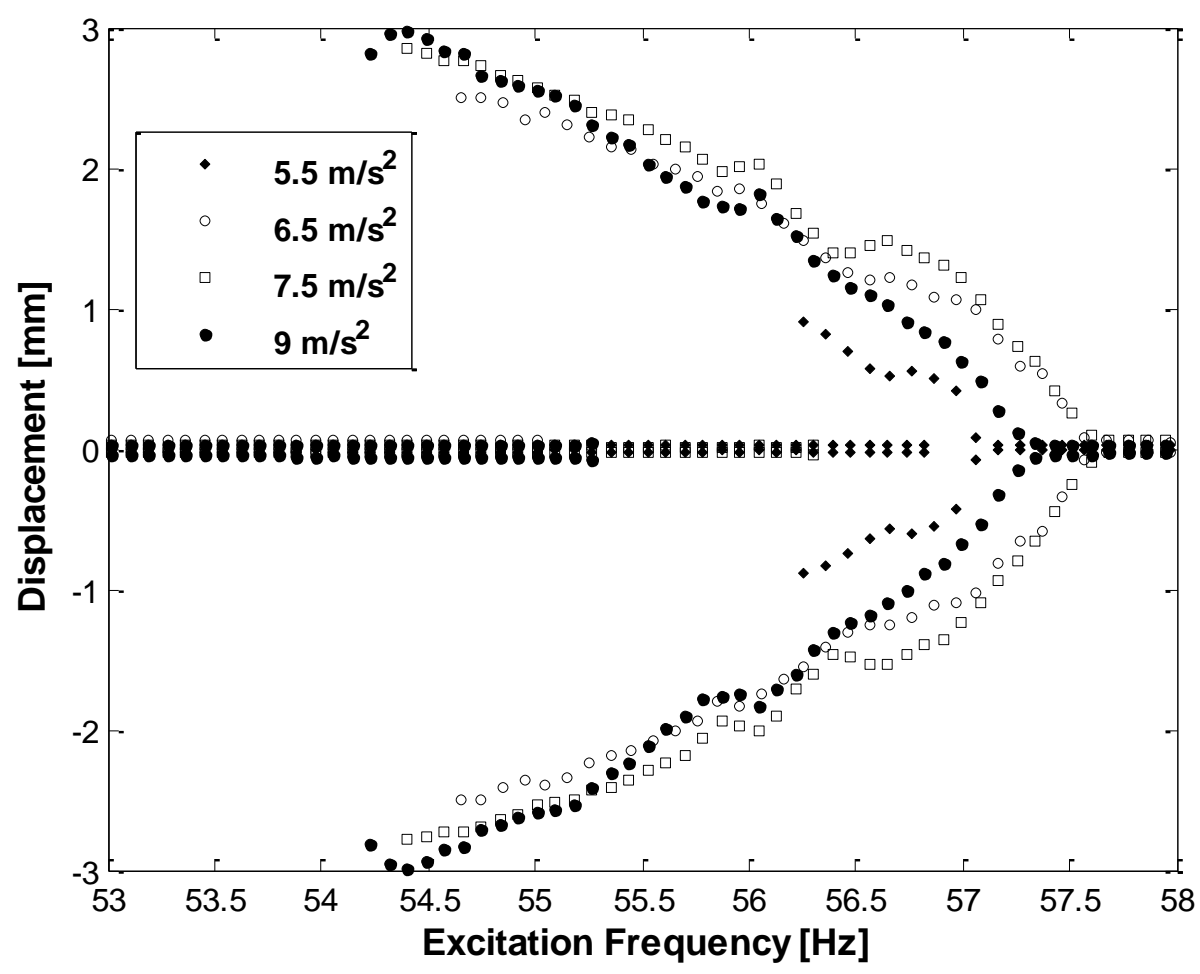

(c)

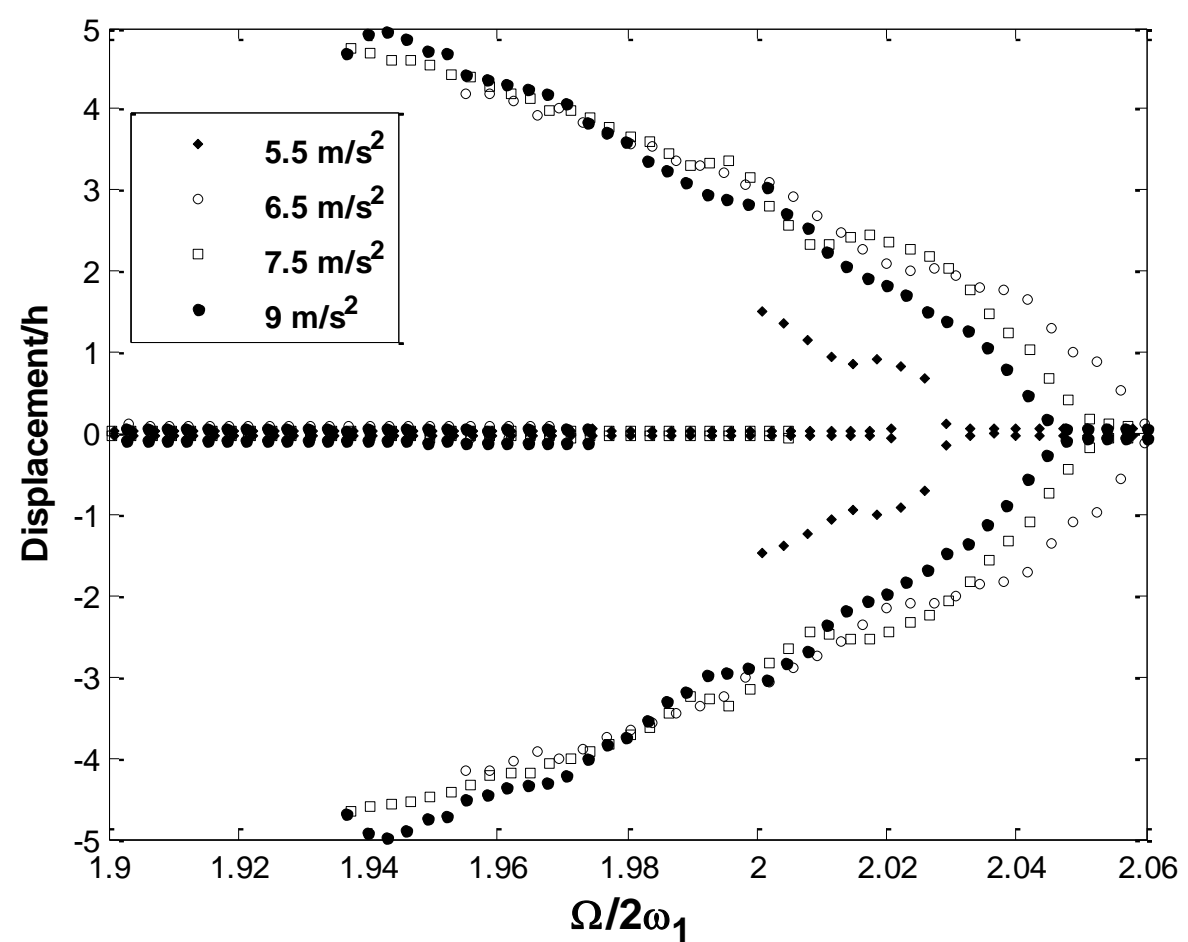


(a)

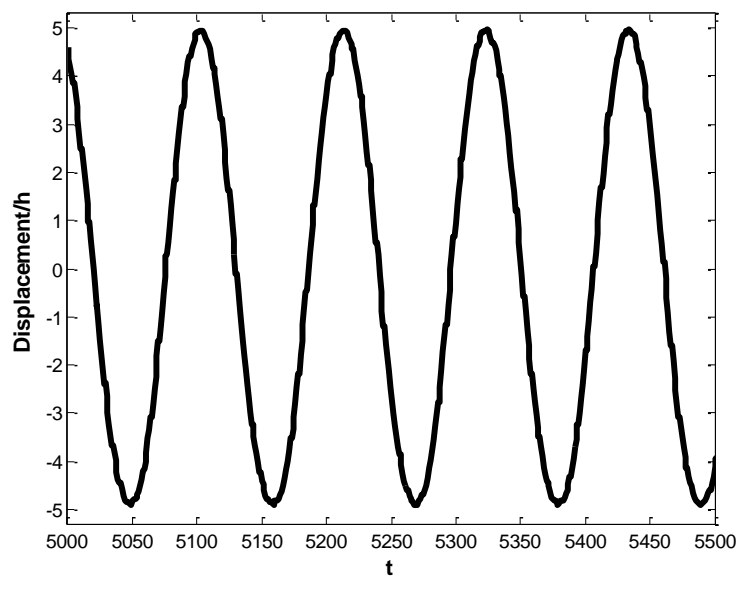

(c)

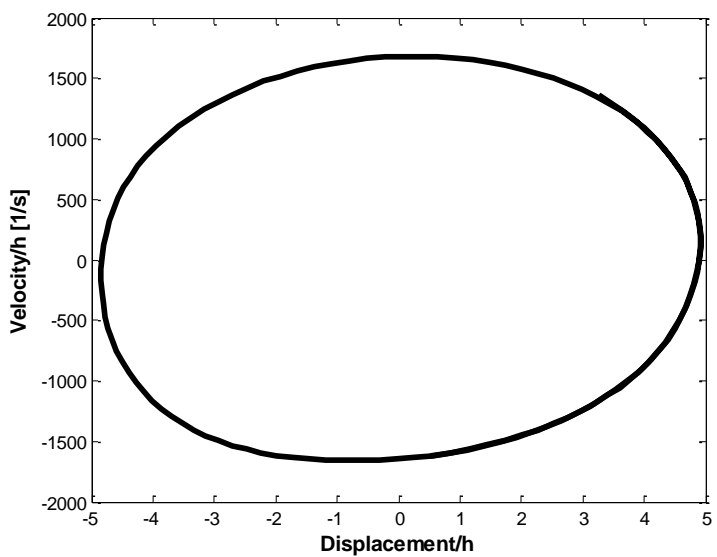

(b)

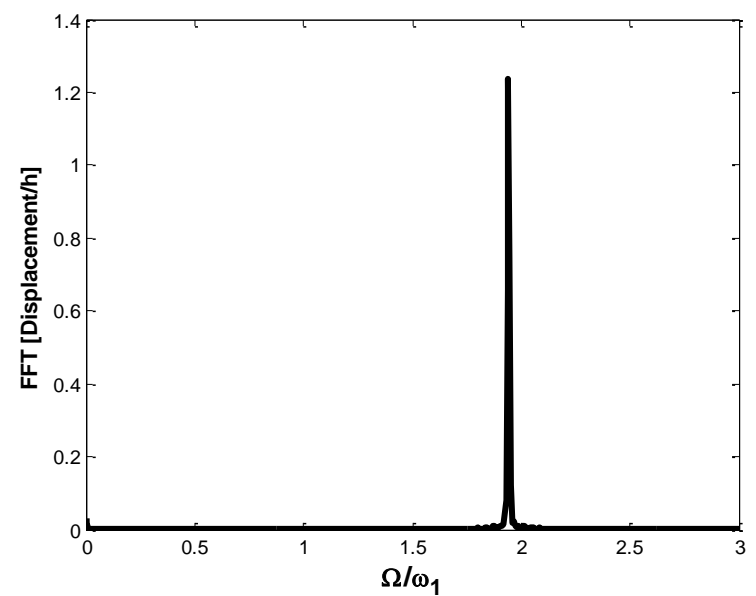

(d)

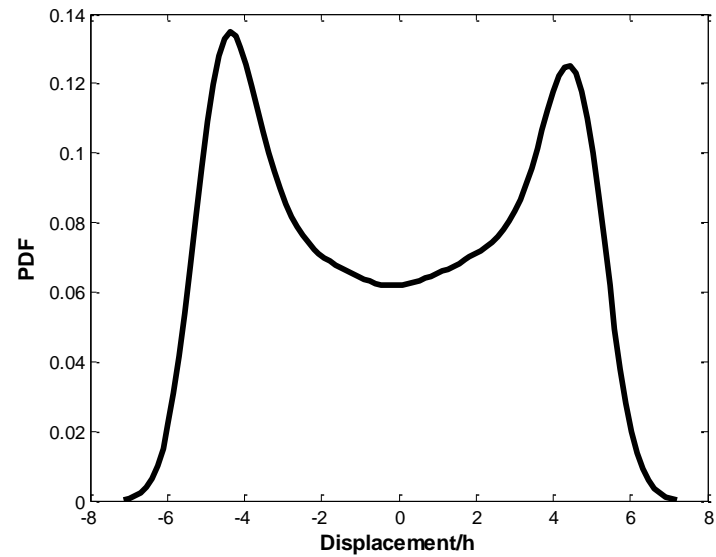

Figure 5: (a) Time trace; (b) FFT; (c) Phase plane diagram; (d) PDF at $\mathbf{\Omega}=54 \mathrm{~Hz}$ and $9 \mathrm{~ms}^{-2}$ base excitation, for the system of Fig. 4, illustrating a period-2 motion

\subsection{Energy harvested from a non-smooth periodic excitation}

In this section, a non-smooth periodic excitation has been generated by the shaker and applied to the base of the energy harvester; the nonlinear energy harvested as well as the nonlinear dynamical response are obtained experimentally and plotted.

Figure 6 illustrates the non-smooth periodic excitation signal from the electrodynamic shaker where Sub-figures 6 (a) and (b) show the time trace and FFT, respectively (there is noise along with a strong periodic component); the input signal is controlled through the accelerometer attached to the shaker (see Figure 2). 
(a)



(b)



Figure 6: (a) Time trace; (b) FFT of input signal from electrodynamic shaker

Figure 7 shows the nonlinear voltage harvested (a), the dimensional nonlinear response (b) and the dimensionless nonlinear response (c); the energy harvester still displays a strong softening-type nonlinearity with discontinuous points occurring in the forward and reverse frequency sweeps at $\Omega=56.36$ and $52.43 \mathrm{~Hz}$, respectively. Comparing Figs. 7 and 4 (with non-smooth and smooth periodic excitations, respectively), the energy harvester performs better for non-smooth periodic excitations than with purely harmonic sinusoids which are more realistic in practical implementation; moreover, the effective operating bandwidth is increased over $20 \%$ and a maximum open-circuit voltage of $0.5 \mathrm{~V}$ is harvested at $52.53 \mathrm{~Hz}$. There are also traces of weak internal resonances present, for instance, in the vicinity of $\Omega=$ 53.94 and $54.55 \mathrm{~Hz}$. The experimentally obtained time trace, FFT, phase-plane diagram and PDF are plotted in Figs.8(a)-(d) for $\Omega=52.52 \mathrm{~Hz}$; the energy harvester displays a period-2 motion which is slightly non-symmetric due to the initial geometric imperfections. 
(a)

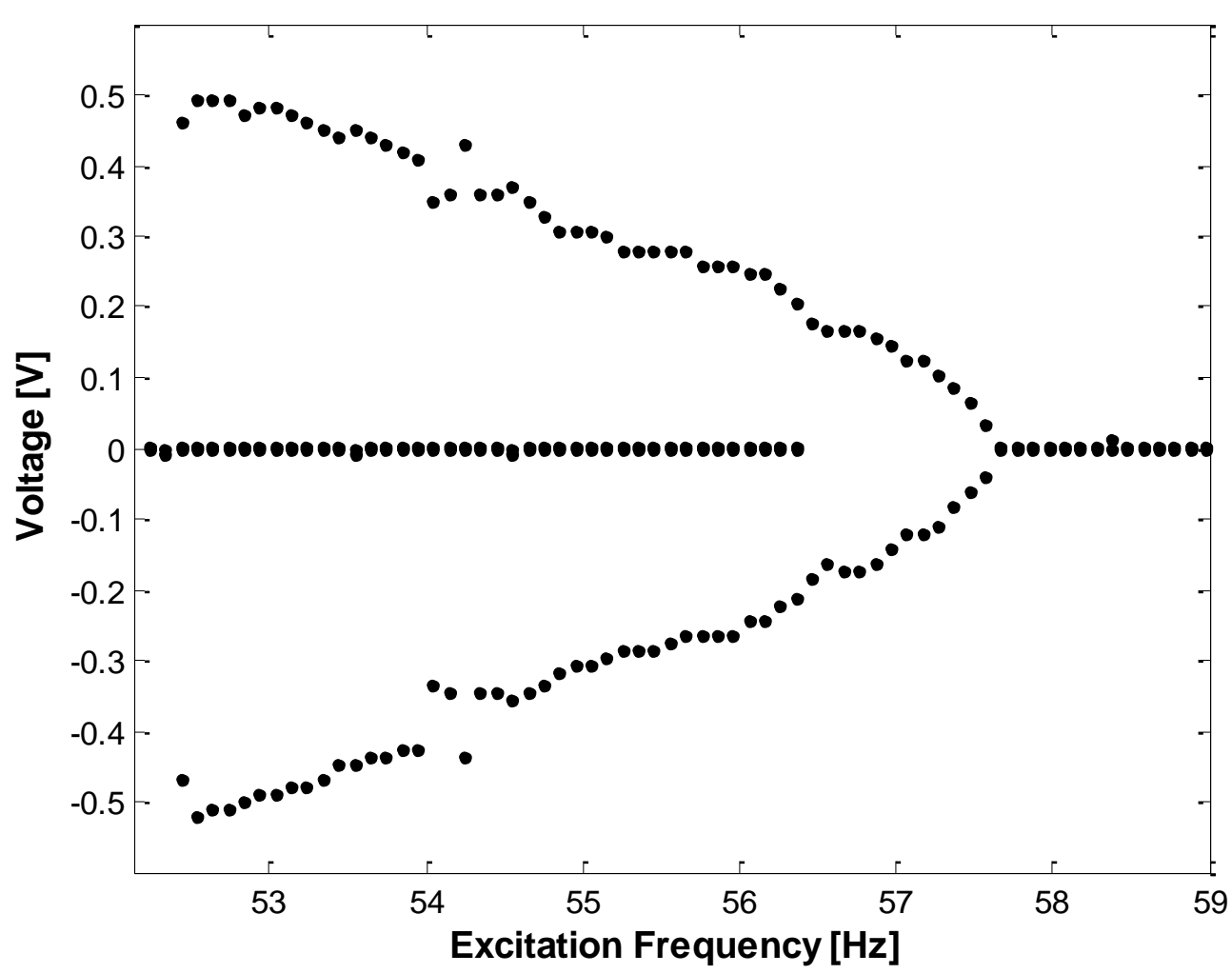

(b)

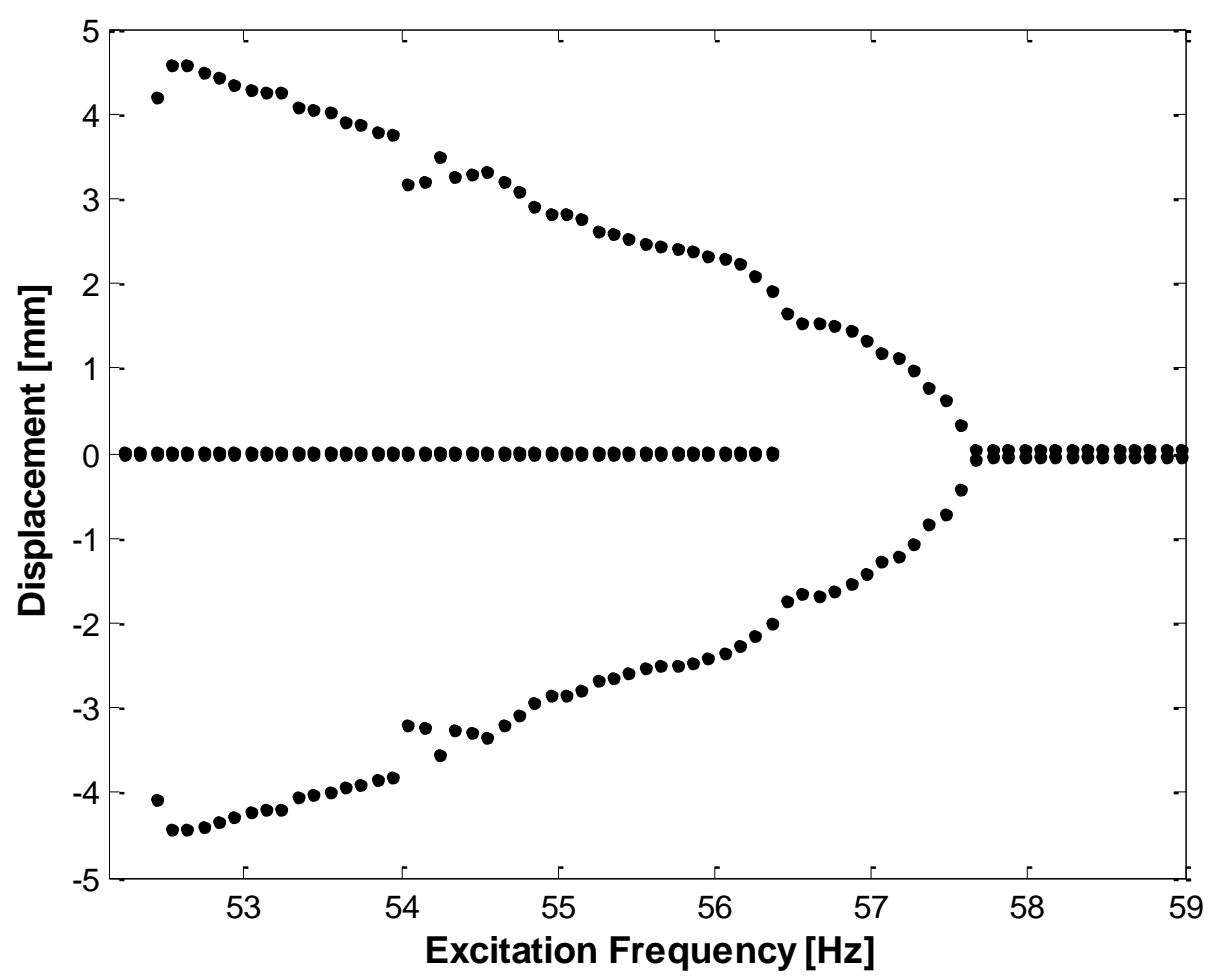


(c)

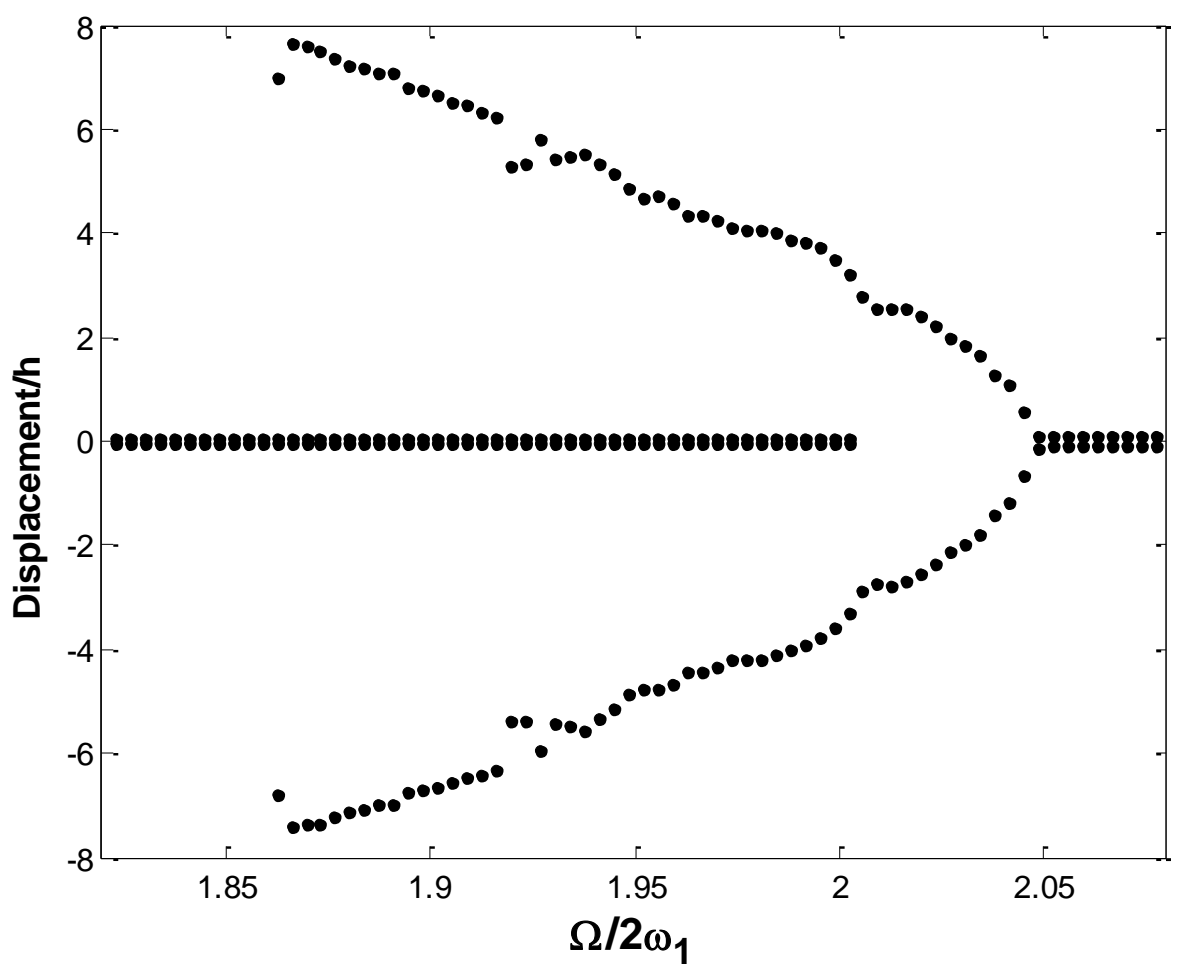
voltage generated; (b) Dimensional displacement; (c) Non-dimensional displacement 
(a)

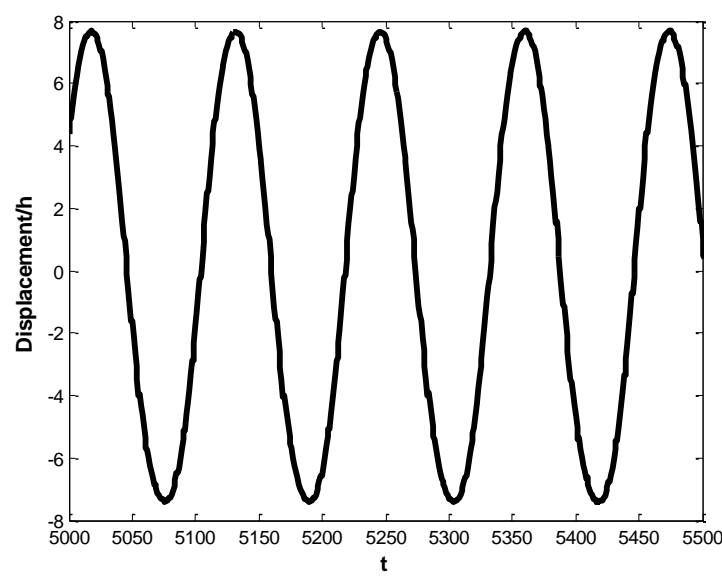

(c)

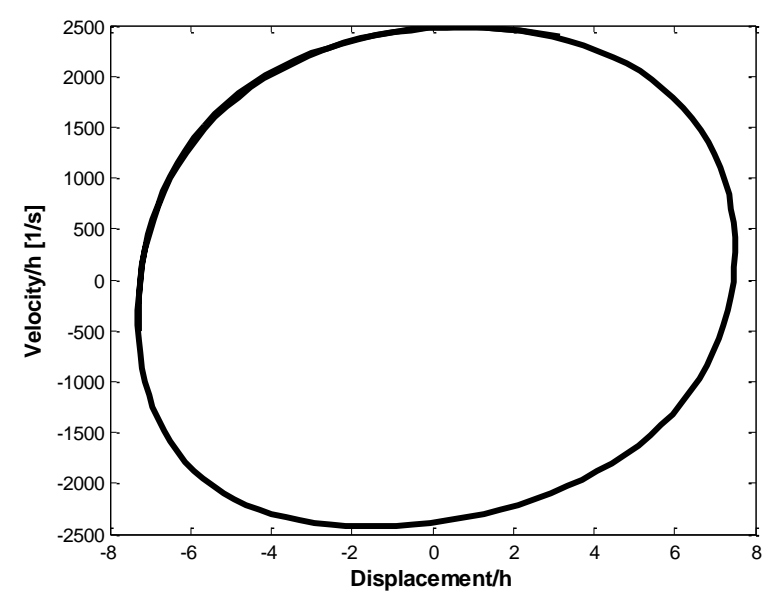

(b)



(d)

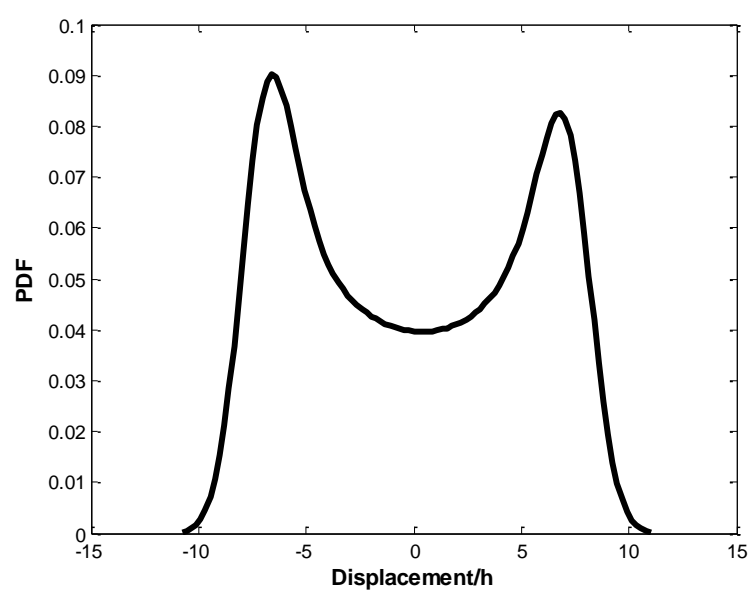

Figure 8: (a) Time trace; (b) FFT; (c) Phase plane diagram; (d) PDF at $\mathbf{\Omega}=52.53 \mathrm{~Hz}$, for the system of Fig. 7, illustrating a period-2 motion

Table 1: Comparison of the excitation types

\begin{tabular}{c|c|c|c} 
Excitation & $\begin{array}{c}\text { Bandwidth } \\
{[\mathbf{H z}]}\end{array}$ & $\begin{array}{c}\text { Max. Open Circuit } \\
\text { Voltage [V] }\end{array}$ & $\begin{array}{c}\text { Max. } \\
\text { Displacement/h }\end{array}$ \\
\hline Harmonic & 3.02 & 0.34 & 4.96 \\
\hline $\begin{array}{c}\text { Non-smooth } \\
\text { periodic }\end{array}$ & 5.24 & 0.52 & 7.624
\end{tabular}

A comparison of the two different excitation types, i.e. harmonic and non-smooth periodic is shown in Table 1. For the comparisons, the harmonic excitation was selected at 9 $\mathrm{m} / \mathrm{s}^{2}$ from Fig. 4; while for the non-smooth periodic excitation case, the system shown in Fig. 
3297 has been used because it has a maximum base excitation of $9 \mathrm{~m} / \mathrm{s}^{2}$ (which is the same as the 330 harmonic case). It was observed that under a non-smooth periodic excitation the fabricated 331 energy harvester has a larger bandwidth of $2.22 \mathrm{~Hz}$ compared to the harmonic excitation 332 case; this result was due to the non-smooth excitation causing more instability of the core 333 element which in turn activates motion attractors which are more beneficial for harvesting energy.

\subsection{Acceleration-response curves}

To further demonstrate the nonlinear parametric behaviour, experiments were also conducted for the acceleration-response curves at a fixed frequency of $56 \mathrm{~Hz}$; this was done as a design requirement to verify the minimum parametric threshold amplitude required to activate a principal parametric resonance as shown in Figure 9. It was observed there are two distinct discontinuous bifurcations at $Y=4.525 \mathrm{~m} / \mathrm{s}^{2}$ and at $Y=5.56 \mathrm{~m} / \mathrm{s}^{2}$; moreover, this behaviour was due to the geometric extensibility at the centreline of the core element, the geometric imperfection and the parametric excitation which have all been used in the design of the fabricated energy harvester to further enhance the operating bandwidth of the device. 
(a)

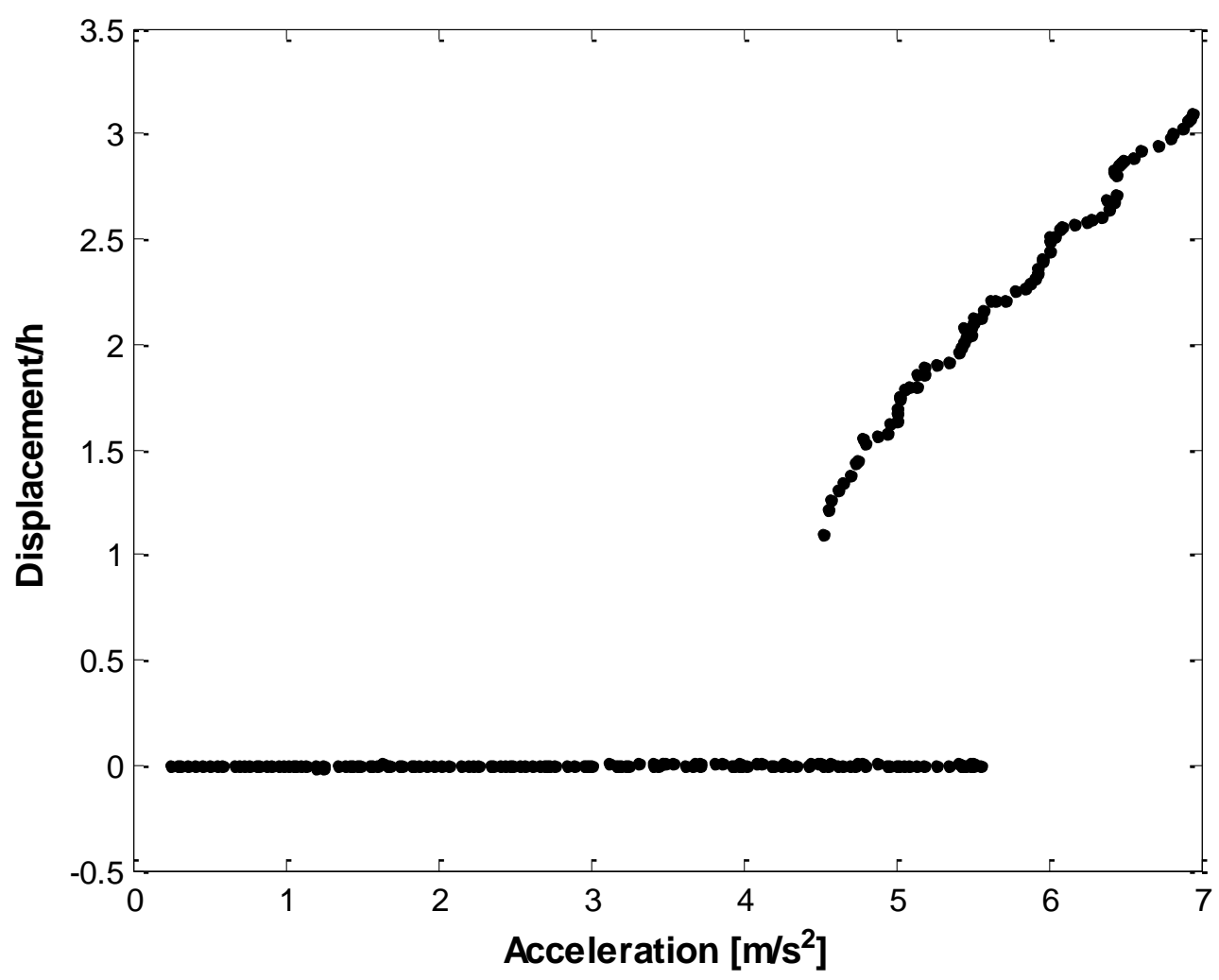

(b)

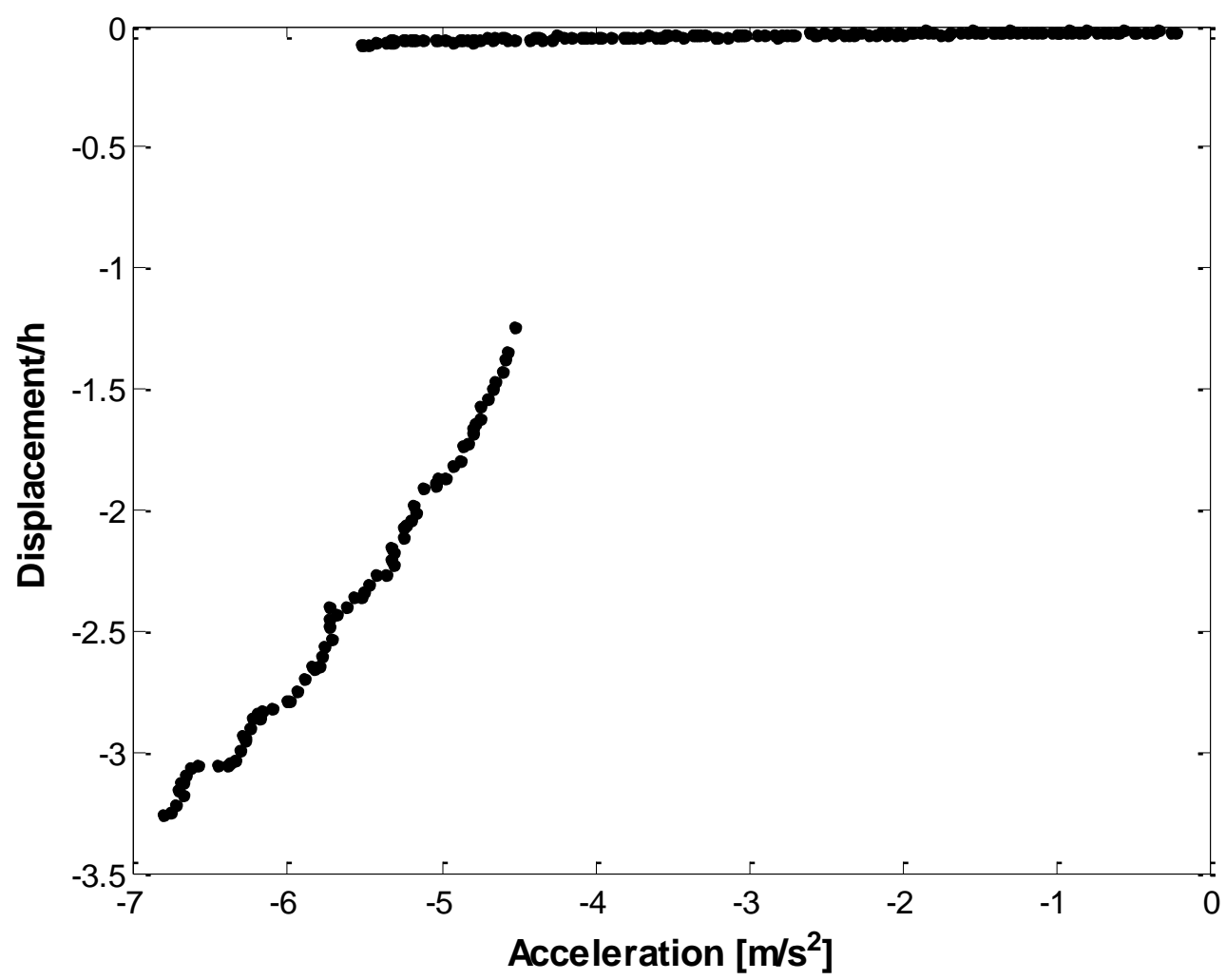
the laser displacement sensor); (b) Negative displacement (i.e. when moving towards the laser displacement sensor) 


\section{Theoretical verification}

In this section, theoretical verifications of the experimentally obtained data has been conducted. COMSOL Multiphysics 5.1, using the solid mechanics module, has been used for the theoretical verifications; a geometry based on the schematic shown in sub-Figure 1 (b) has been developed-moreover, the geometry has been meshed using 375907 elements. The theoretically calculated primary resonance of the energy harvester was $29.687 \mathrm{~Hz}$ while the experimentally obtained natural frequency was $28.27 \mathrm{~Hz}$; furthermore, the theoretical parametric resonance was calculated to be $59.374 \mathrm{~Hz}$ while the experimentally obtained parametric was in the vicinity of $57.5 \mathrm{~Hz}$ - these results are within very good agreement of each other. From theoretical simulations the maximum parametric motion amplitude occurred at the centre of the beam as shown in Figure 10.

Figure 10: Theoretical mode shape of the fundamental and parametric resonance

Theoretical simulations for the frequency-response curves have also been done using

COMSOL Multiphysics to verify the experimentally obtained results at the fundamental 

results was obtained - this was due to the axial excitation resulting in a zero-response experimentally, however, results are within good agreement.

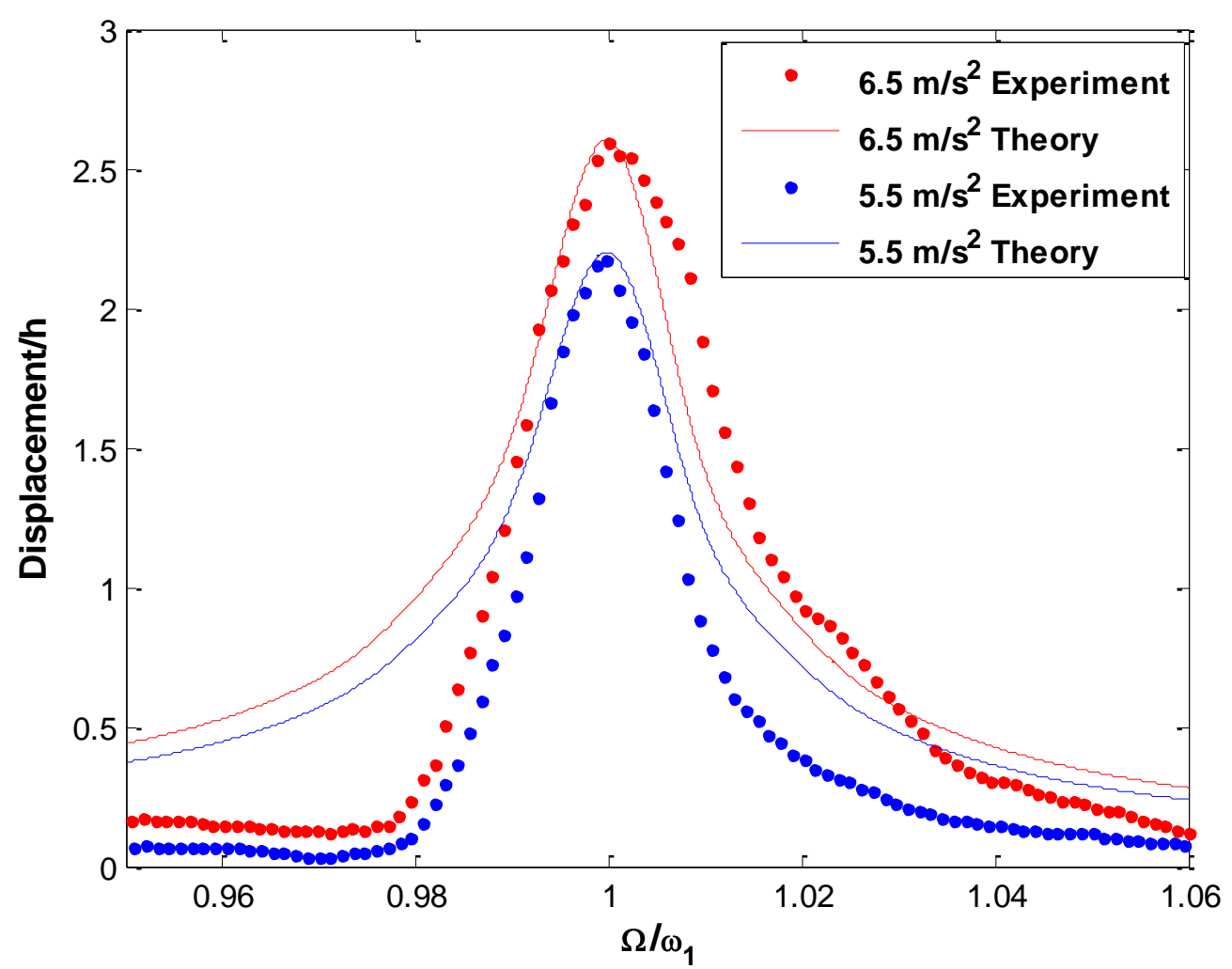

Figure 11: Comparison between theoretical and experimental results obtained at the fundamental resonance

\section{Conclusions}

An energy harvester has been designed, fabricated and tested, operating based on the nonlinear dynamical response of a parametrically excited clamped-clamped system using a

377 beam with a point-mass (magnets) as the core element; as the core element resonates, the magnets pass through a coil to generate a backward electromotive force for energy harvesting. An electrodynamic shaker was used to excite the energy harvester and experiments have been conducted when the core element was excited (i) harmonically near the primary resonance; (ii) harmonically in the vicinity of the principal parametric resonance; (iii) in the vicinity of the principal parametric resonance with a non-smooth periodic 
excitation. Experiments (i-iii) were used to evaluate the increased operating bandwidth of the nonlinear energy harvester.

The magnets attached to the core element showed non-symmetric motion (hence energy harvested) due to a small initial geometric imperfection; the parametric displacement was larger in one direction about the initial equilibrium position. It was observed that when the energy harvester was excited near the primary resonance, linear energy was harvested. When the core element was excited in the vicinity of the principal parametric resonance, the dynamics substantially changed; a strong softening-type nonlinearity was observed near $\Omega / 2 \omega_{1}$ which was due to the effects of parametric excitations, nonlinearity and initial geometric imperfection about the equilibrium position. In the vicinity of the principal parametric resonance, the nonlinear energy harvested possesses two discontinuities (i.e. saddle-node and period-doubling bifurcations); this nonlinear behaviour is advantageous in broadening the frequency range at which the energy is harvested - the motion amplitude was also larger than its linear counterpart at the primary resonance. With a non-smooth periodic excitation in the vicinity of the principal parametric resonance, the system performed best, displaying the largest motion amplitude and the energy harvested as well as effective operating bandwidth; the energy harvester displayed a period-2 motion. For the theoretical investigation, the calculated fundamental and parametric resonances are within very good agreement of each other; moreover, the theoretical frequency-response curves are also within good agreement of the experimental results.

It was observed that the system harvests energy at both the primary and principal parametric resonances (displaying strong softening-type nonlinearity at the principal parametric resonance); the parametric design has substantial qualitative and quantitative effects on the nonlinear dynamical response, hence increasing the amount of energy harvested and effective operating bandwidth of the device. 
1. Saadon, S. and O. Sidek, A review of vibration-based MEMS piezoelectric energy harvesters. Energy Conversion and Management, 2011. 52(1): p. 500-504.

2. Adhikari, S., M.I. Friswell, and D.J. Inman, Piezoelectric energy harvesting from broadband random vibrations. Smart Materials and Structures, 2009. 18(11).

3. Renno, J.M., M.F. Daqaq, and D.J. Inman, On the optimal energy harvesting from a vibration source. Journal of Sound and Vibration, 2009. 320(1-2): p. 386-405.

4. Fan, K.-Q., et al., Design and experimental verification of a bi-directional nonlinear piezoelectric energy harvester. Energy Conversion and Management, 2014. 86: p. 561-567.

5. Fan, K., et al., Design and development of a multipurpose piezoelectric energy harvester. Energy Conversion and Management, 2015. 96: p. 430-439.

6. Guan, M. and W.-H. Liao, Design and analysis of a piezoelectric energy harvester for rotational motion system. Energy Conversion and Management, 2016. 111: p. 239-244.

7. $\mathrm{Bu}$, L., et al., Non-resonant electrostatic energy harvester for wideband applications. IET Micro Nano Letters, 2013. 8(3): p. 135-137.

8. Sardini, E. and M. Serpelloni, An efficient electromagnetic power harvesting device for lowfrequency applications. Sensors and Actuators A: Physical, 2011. 172(2): p. 475-482.

9. Marin, A., et al., Broadband electromagnetic vibration energy harvesting system for powering wireless sensor nodes. Smart Materials and Structures, 2013. 22(7).

10. Ooi, B.L. and J.M. Gilbert, Design of wideband vibration-based electromagnetic generator by means of dual-resonator. Sensors and Actuators A: Physical, 2014. 213: p. 9-18.

11. Siddique, A.R.M., S. Mahmud, and B.V. Heyst, A comprehensive review on vibration based micro power generators using electromagnetic and piezoelectric transducer mechanisms. Energy Conversion and Management, 2015. 106: p. 728-747.

12. Roundy, S. and Y. Zhang. Toward self-tuning adaptive vibration-based microgenerators. in Proc. SPIE. 2005.

13. Williams, C.B. and R.B. Yates, Analysis of a micro-electric generator for microsystems. Sensors and Actuators A: Physical, 1996. 52(1-3): p. 8-11.

14. Mitcheson, P.D., et al., Architectures for vibration-driven micropower generators. Journal of Microelectromechanical Systems, 2004. 13(3): p. 429-440.

15. Stephen, N.G., On energy harvesting from ambient vibration. Journal of Sound and Vibration, 2006. 293(1-2): p. 409-425.

16. Shahruz, S.M., Design of mechanical band-pass filters with large frequency bands for energy scavenging. Mechatronics, 2006. 16(9): p. 523-531.

17. Tang, X. and L. Zuo, Enhanced vibration energy harvesting using dual-mass systems. Journal of Sound and Vibration, 2011. 330(21): p. 5199-5209.

18. Erturk, A. and D.J. Inman, On mechanical modeling of cantilevered piezoelectric vibration energy harvesters. Journal of Intelligent Material Systems and Structures, 2008. 19(11): p. 1311-1325.

19. Leland, E.S. and P.K. Wright, Resonance tuning of piezoelectric vibration energy scavenging generators using compressive axial preload. Smart Materials and Structures, 2006. 15(5).

20. Eichhorn, C., F. Goldschmidtboeing, and P. Woias, Bidirectional frequency tuning of a piezoelectric energy converter based on a cantilever beam. Journal of Micromechanics and Microengineering, 2009. 19(9).

21. Mann, B.P. and N.D. Sims, Energy harvesting from the nonlinear oscillations of magnetic levitation. Journal of Sound and Vibration, 2009. 319(1-2): p. 515-530.

22. Maiorca, F., et al., Diode-less mechanical H-bridge rectifier for "zero threshold" vibration energy harvesters. Sensors and Actuators A: Physical, 2013. 201: p. 246-253. 
23. Liu, H., et al., Investigation of a MEMS piezoelectric energy harvester system with a frequency-widened-bandwidth mechanism introduced by mechanical stoppers. Smart Materials and Structures, 2012. 21(3).

24. Sebald, G., et al., Experimental Duffing oscillator for broadband piezoelectric energy harvesting. Smart Materials and Structures, 2011. 20(10).

25. Masana, R. and M.F. Daqaq, Response of duffing-type harvesters to band-limited noise. Journal of Sound and Vibration, 2013. 332(25): p. 6755-6767.

26. Halvorsen, E., Fundamental issues in nonlinear wideband-vibration energy harvesting. Physical Review E - Statistical, Nonlinear, and Soft Matter Physics, 2013. 87(4).

27. Abdelkefi, A., A.H. Nayfeh, and M.R. Hajj, Global nonlinear distributed-parameter model of parametrically excited piezoelectric energy harvesters. Nonlinear Dynamics, 2012. 67(2): p. 1147-1160.

28. Daqaq, M.F., et al., Investigation of power harvesting via parametric excitations. Journal of Intelligent Material Systems and Structures, 2009. 20(5): p. 545-557.

29. Mann, B.P. and N.D. Sims, On the performance and resonant frequency of electromagnetic induction energy harvesters. Journal of Sound and Vibration, 2010. 329(9): p. 1348-1361.

30. Elvin, N.G. and A.A. Elvin, An experimentally validated electromagnetic energy harvester. Journal of Sound and Vibration, 2011. 330(10): p. 2314-2324. 